ANL-HEP-PR-96-33

MC-TH-96-16

\title{
New Parton Distribution Functions for the Photon
}

\author{
L. E. Gordon ${ }^{a}$ and J. K. Storrow ${ }^{b}$ \\ ${ }^{a}$ High Energy Physics Division, Argonne National Laboratory, Argonne, IL 60439, USA \\ ${ }^{b}$ Dept. of Theoretical Physics, University of Manchester, Manchester M13 9PL, England
}

\begin{abstract}
We present new improved parton distributions for the photon. We fit all available data on the photon structure function, $F_{2}^{\gamma}\left(x, Q^{2}\right)$, with $Q^{2} \geq 3$ $\mathrm{GeV}^{2}$, in order to determine the quark distributions. We also pay particular attention to the gluon distribution in the photon, $g^{\gamma}\left(x, Q^{2}\right)$, which has been poorly constrained in earlier analyses which only include structure function data. We use large $p_{T}$ jet production in $\gamma \gamma$ collisions from TRISTAN to constrain $g^{\gamma}$. We also see what information can be gleaned from $\gamma p$ collisions at HERA on $g^{\gamma}$ and on the quark distributions at large $x$, where there are no structure function data. We review future prospects of elucidating the parton distributions of the photon.
\end{abstract}

Typeset using REVTEX 


\section{INTRODUCTION}

Some time ago, we presented some parton distribution functions (pdfs) for the photon [1]. These distributions, which we will refer to here as the GS distributions, were the first available in both leading order(LO) and next-to-leading order(NLO) QCD. Since then they have been used as input to perturbative QCD (PQCD) comparisons to a variety of types of data with a fair degree of success. Applications in $\gamma \gamma$ collisions at various $e^{+} e^{-}$colliders are not restricted to the analysis of $F_{2}^{\gamma}\left(x, Q^{2}\right)$ data [2]; they also include the inclusive production of jets [3] [6] and single hadrons at large $p_{T}$ [7, 8]. Applications at the ep collider HERA include the production of jets and dijets [9] 13] and single hadrons [7, 14, 15] at large $p_{T}$. Up to now, no contradiction with any data has been found. These pdfs have also been used in estimates for rarer processes such as the photoproduction of high-mass di-lepton pairs [16] and large $p_{T}$ prompt photons 17,18.

At that time, and up until fairly recently, the only experimental information on the pdfs of the photon was obtained from studies of the structure function of the photon, $F_{2}^{\gamma}\left(x, Q^{2}\right)$, in two photon collisions at $e^{+} e^{-}$colliders. In $\mathrm{LO} \mathrm{QCD}, F_{2}^{\gamma}\left(x, Q^{2}\right)$ is related to the quark and anti-quark distributions in the photon, $q_{i}^{\gamma}\left(x, Q^{2}\right)$ and $\bar{q}_{i}^{\gamma}\left(x, Q^{2}\right)$ respectively, by,

$$
F_{2}^{\gamma}\left(x, Q^{2}\right)=\sum_{i=1}^{N_{f}} x e_{i}^{2}\left(q_{i}^{\gamma}\left(x, Q^{2}\right)+\bar{q}_{i}^{\gamma}\left(x, Q^{2}\right)\right)
$$

and thus the quark distributions are fairly directly determined from the data on $F_{2}^{\gamma}\left(x, Q^{2}\right)$. Theoretically, this is a very interesting area, because, at large $x$ and asymptotically large $Q^{2}$, the pdfs of the photon and hence $F_{2}^{\gamma}\left(x, Q^{2}\right)$ are predicted from PQCD in both LO 19 and NLO 20,21. The so-called 'anomalous' piece is calculable as a function of $x$ and $Q^{2}$ and it is the $Q^{2}$ dependence (specifically $\propto\left(\alpha_{s}\left(Q^{2}\right)\right)^{-1}$ ) that ensures that it dominates at asymptotic values of $Q^{2}$. However, it is singular as $x \rightarrow 0$ and to regularise these singularities, some non-perturbative input to the Altarelli-Parisi(AP) equations is required, at a reference $Q^{2}=Q_{0}^{2}$. The anomalous $\left(\alpha_{s}\left(Q^{2}\right)\right)^{-1}$ behaviour of the pdfs arises because of the direct $\gamma \rightarrow q \bar{q}$ coupling, which gives inhomogeneous terms in the AP equations [22]: this 
is the special feature of the photon. However, in the $Q^{2}$ range experimentally accessible at present and in the foreseeable future, the effect of this input at a $Q^{2}=Q_{0}^{2}$ is significant, and so the analysis of the photon structure function is similar to that of a hadronic structure function. Different groups make different choices of input scale $Q_{0}^{2}$, different forms of input pdfs, and all fit parameters to $F_{2}^{\gamma}\left(x, Q^{2}\right)$ data at various $Q^{2}$. The different possibilities have been recently reviewed in ref [23].

The gluon distribution of the photon, $g^{\gamma}\left(x, Q^{2}\right)$, are not well constrained by these structure functions analyses [1], essentially because the coupling of the AP equations for the gluon and singlet quark sectors is weak, and so the output (evolved) quark pdfs and thus the evolved values of $F_{2}^{\gamma}\left(x, Q^{2}\right)$ (for $Q^{2} \geq Q_{0}^{2}$ ) are rather independent of the input gluon distribution at $Q^{2}=Q_{0}^{2}, g^{\gamma}\left(x, Q_{0}^{2}\right)$. Furthermore, and even more importantly, unlike the case for hadrons, $g^{\gamma}$ is not constrained by a momentum sum rule [1, 24]. The result of all this is that the available parametrisations of the photon distributions agree reasonably well in their quark distributions but can have considerably different gluon distributions 2325. The input gluon distributions in the evolution equations, while not completely arbitrary, are currently just theoretically motivated guesses.

Since our distributions were published, there has been much experimental activity in this and related areas. Not only has the amount of data on $F_{2}^{\gamma}$ been greatly increased, but data on jet production in both $\gamma \gamma$ collisions at $e^{+} e^{-}$colliders and in $\gamma p$ collisions at HERA have appeared. These provide information on both quark and gluon distributions in the photon via resolved photon processes [26], as we shall see.

In view of this and also in view of the fact that there is expected to be an explosion of data in all of these areas in the next few years, it seems like an opportune moment to provide improved versions of our pdfs. The new data will come from higher luminosity running at HERA and also the commissioning of LEP2, which is anticipated to provide several hundreds of inverse pb of integrated luminosity in $e^{+} e^{-}$collisions over its lifetime. The improvements we anticipate making are as follows:

(1) Extending the kinematic range of validity in $Q^{2}$ from $Q^{2} \geq 5.3 \mathrm{GeV}^{2}$ down to $Q^{2} \geq 3$ 
$\mathrm{GeV}^{2}$.

(2) Fitting to all available data on $F_{2}^{\gamma}$ to obtain a better determination of the quark distributions.

(3) Constraining the input gluon distribution by fitting the TRISTAN data on jet production in $\gamma \gamma$ collisions. We will also see what can be learned from HERA data on jet production.

The plan of this paper is as follows. In subsect. II.A we discuss general considerations about the pdfs of the photon. In subsect. II.B, we set up the parametrisation of the LO pdfs and in subsect. II.C we discuss our prescription for going over to the NLO parametrisation. In both cases we pay particular attention to the large $x$ behaviour. In sect. III we present our fits to the data, considering separately the data on $F_{2}^{\gamma}$ (subsect. III.A), jets in $\gamma \gamma$ collisions (subsect. III.B), and jets in $\gamma p$ collisions (subsect. III.C). In sect. IV we discuss the properties of the new distributions and review future prospects for improving our knowledge of the photon pdfs. In sect. $\mathrm{V}$ we present conclusions.

\section{NEW PHOTON DISTRIBUTIONS}

\section{A. General Considerations}

To obtain pdfs for the photon, we must start with input photon pdfs at some reference scale $Q_{0}^{2}$, and choosing an appropriate value of $Q_{0}^{2}$ provides the first problem. Making a vector meson dominance (VMD) ansatz seems an obvious starting point as VMD provides a connection between the photon and $\rho$ meson pdfs. If we then use $\mathrm{SU}(6)$ to relate the $\rho$ pdfs to those of the pion, we can then use experimental constraints on the pion pdfs from Drell-Yan lepton pair and prompt photon production data [27. In such a VMD ansatz, the gluon is fixed by a momentum sum rule via a mesonic momentum sum rule. However, a problem arises here: if we choose a plausible VMD scale, $Q_{0}^{2} \simeq 1 \mathrm{GeV}^{2}$, a pure VMD input is known to be insufficient to fit the data at higher $Q^{2}$ [28 30]. To circumvent this problem 
two separate approaches have been adopted. The first is to maintain the VMD approach but to start the AP evolution at a very low scale significantly below $Q_{0} \simeq 1 \mathrm{GeV} 31$ [34]. The other is to take an input scale significantly above $Q_{0} \simeq 1 \mathrm{GeV}$ and fit the quark pdfs to the $F_{2}^{\gamma}$ data here, which essentially means supplementing the VMD input with a point-like component, seemingly naturally provided by the Born-Box diagram [1, 34 37]. Unfortunately there is no correspondingly natural choice for the gluon density and a guess must be made. This is the approach that we will adopt here, as we did in ref [1], though we will be reducing from the value chosen there, $Q_{0}^{2}=5.3 \mathrm{GeV}^{2}$, to $Q_{0}^{2}=3 \mathrm{GeV}^{2}$. In ref [1] we advocated choosing the gluon content to ensure that the ratio of momentum carried by the quarks and antiquarks relative to that carried by the gluons, $R_{2}\left(Q^{2}\right)$, should lie between 1 and 3 . The motivation for these limits is as follows. At low $Q^{2}$ we expect VMD to be good, the photon to be hadronic, and $R_{2} \simeq 1$ as for a hadron: at high $Q^{2}$, we expect the asymptotic result of PQCD [38], that $R_{2}=99 / 32 \simeq 3$ to hold, and so we expect a steady increase from 1 to 3 as $Q^{2}$ increases. It is interesting that at the time we noted that the LAC distributions have such a large gluon component that they are in danger of violating this bound. Since then LAC3 has been rejected by the data, because the large gluon component at large $x$ overestimates the jet cross section in both $\gamma \gamma$ and $\gamma p$ collisions and LAC1 seems to have too many gluons at small $x$ compared to the $\mathrm{H} 1$ data. We will expand on this later.

\section{B. Input Distributions: LO case}

To obtain regularised distributions in LO we separate the photon structure function into a hadronic and a point-like part [28]

$$
F_{2}^{\gamma}\left(x, Q_{0}^{2}\right)=F_{2}^{P L}\left(x, Q_{0}^{2}\right)+F_{2}^{H A D}\left(x, Q_{0}^{2}\right)
$$

The hadronic part, which we assume can be approximated by VMD, is important at small values of $x(\leq 0.2)$ while the point-like part, which we base on the Quark Parton Model (QPM) formula, models the medium to large $x$ part. 
The hadronic part of the input was chosen according to standard VMD ideas. We assume that the hadronic photon can be represented by the $\rho^{0}$ meson

$$
F_{2, V M D}^{\gamma}\left(x, Q^{2}\right)=\frac{4 \pi \alpha_{e m}}{f_{\rho}^{2}} \sum_{i} e_{i}^{2} x q_{i}^{\rho^{o}}\left(x, Q^{2}\right)
$$

where $f_{\rho}^{2} / 4 \pi \approx 2.2$, and use $\mathrm{SU}(6)$ and isospin invariance to relate the $\rho^{0}$ distributions to those of the charged pions which are constrained by data [27]:

$$
q_{i}^{\rho^{o}}\left(x, Q_{0}^{2}\right)=q_{i}^{\pi^{0}}(x)=\frac{1}{2}\left(q_{i}^{\pi^{+}}(x)+q_{i}^{\pi^{-}}(x)\right)
$$

The above assumptions are supported by the fact that the VMD estimates of the structure function $F_{2}^{\gamma}\left(x, Q^{2}\right)$ base agree with the data at $Q^{2}=1 \mathrm{GeV}^{2}$ [49,50]. We also make the plausible assumption that the VMD breakdown into singlet, non-singlet and gluon sectors is also reliable. In terms of valence $v^{\pi}(x)$ and sea, $\zeta^{\pi}(x)$, these are given by

$$
\begin{aligned}
q_{N S, V M D}^{\gamma}\left(x, Q_{0}^{2}\right) & =\kappa \frac{4 \pi \alpha_{e m}}{f_{\rho}^{2}}\left(\frac{1}{9} v^{\pi}\right) \\
\Sigma_{V M D}^{\gamma}\left(x, Q_{0}^{2}\right) & =\kappa \frac{4 \pi \alpha_{e m}}{f_{\rho}^{2}}\left(2 v^{\pi}+6 \zeta^{\pi}\right) \\
G_{V M D}^{\gamma}\left(x, Q_{0}^{2}\right) & =\kappa \frac{4 \pi \alpha_{e m}}{f_{\rho}^{2}} G^{\pi}(x)
\end{aligned}
$$

where we have used $N_{f}=3$ flavours since the heavy quark contributions are expected to be very small at this $Q^{2}$. The constant $\kappa$, where $1 \leq \kappa \leq 2$, was introduced in ref. 39 to represent the uncertainty over the inclusion of higher mass vector mesons. We take the following forms [1]:

$$
\begin{aligned}
& x v^{\pi}(x)=A \sqrt{x}(1-x) \\
& x \zeta^{\pi}(x)=B(1-x)^{5} \\
& x G^{\pi}(x)=C(1-x)^{3}
\end{aligned}
$$

The conservation of baryon number fixes $A$ at 0.75 , fixing the momentum fraction of the hadronic part carried by the valence sector at $40 \%$. The momentum fractions carried by the sea and gluon sectors are fixed by $B$ and $C$ which we use, along with $\kappa$ as free parameters in our fits to the $F_{2}^{\gamma}$ data. 
For the point-like part we use the lowest order Bethe-Heitler (B-H) form 40,41]

$$
\begin{aligned}
q_{i}^{\gamma}\left(x, Q^{2}\right)= & 3 e_{i}^{2} \frac{\alpha_{e m}}{\pi}\left[\beta \left(\left(8 x(1-x)-1-\frac{4 m_{i}^{2}}{Q^{2}} x(1-x)\right)\right.\right. \\
& \left.+\left(x^{2}+(1-x)^{2}+\frac{4 m_{i}^{2}}{Q^{2}} x(1-3 x)-\frac{8 m_{i}^{4}}{Q^{4}} x^{2}\right) \ln \left(\frac{1+\beta}{1-\beta}\right)\right]
\end{aligned}
$$

for $\beta^{2} \geq 0$ where

$$
\beta^{2}=1-\frac{4 m_{i}^{2} x}{(1-x) Q^{2}}
$$

and $m_{i}$ are the light quark masses which are taken as fairly closely constrained parameters. Now $q_{i}^{\gamma}\left(x, Q^{2}\right)=0$ for $\beta^{2} \leq 0$, i.e. for

$$
1 \geq x \geq x_{i}=\frac{Q^{2}}{Q^{2}+4 m_{i}^{2}}
$$

and typically $x_{i} \simeq 0.9$ for light quarks and $Q_{0}^{2}=3 \mathrm{GeV}^{2}$. Also the VMD piece vanishes as $x \rightarrow 1$ as a power of $(1-x)$. Hence our quark distributions will be greatly suppressed at large $x$, a point we will return to later. The parametric form for the distributions are thus

$$
q_{i}^{\gamma}\left(x, Q_{0}^{2}\right)=q_{i}^{P L}\left(x, m_{u}, m_{s}\right)+q_{i}^{H A D}(x, \kappa, B, C)
$$

for the singlet and non-singlet sectors and for the gluon we take the form:

$$
g^{\gamma}\left(x, Q_{0}^{2}\right)=g(x, \kappa, C)+\frac{2}{\beta_{0}} \lambda P_{g q}(x) * \Sigma\left(x, m_{i}\right)
$$

and the convolution $*$ is defined by

$$
A(x) * B(x)=\int_{x}^{1} \frac{d y}{y} A(x / y) B(y)
$$

and $\lambda$ is a parameter which enables us to adjust the component of the gluon input estimated from bremsstrahlung off the singlet quarks. This will increase the fraction of the photon momentum carried by the gluons, and compensate for the increase in the momentum carried by the quarks due to inclusion of the point-like contribution. In our earlier work [1], where we took $Q_{0}^{2}=5.3 \mathrm{GeV}^{2}$, we presented two distributions, GS(I) and GS(II), corresponding to $\lambda=1,0$ respectively: these numbers cannot be compared directly with those in the present work, as a different value of $Q_{0}^{2}$ is used. 


\section{Input Distributions: NLO case}

The input distributions in NLO were obtained from the $\mathrm{LO}$ ones by demanding that the structure function, $F_{2}^{\gamma}\left(x, Q_{0}^{2}\right)$, be the same at $Q_{0}^{2}=3 \mathrm{GeV}^{2}$ in LO and NLO i.e.

$$
F_{2}^{\gamma, N L O}\left(x, Q_{0}^{2}\right)=F_{2}^{\gamma, L O}\left(x, Q_{0}^{2}\right)
$$

in the same way as in ref. [四]. For the gluons we simply take

$$
g^{\gamma, N L O}\left(x, Q_{0}^{2}\right)=g^{\gamma, L O}\left(x, Q_{0}^{2}\right)
$$

but choosing the NLO quark distributions in order to satisfy the above condition is a nontrivial procedure which highlights the well-known fact that pdfs are renormalisation scheme dependent and have no physical significance on their own.

To proceed, we assume that the distributions in NLO can be obtained from the LO ones by the addition of a correction term

$$
q_{i}^{\gamma, N L O}\left(x, Q_{0}^{2}\right)=q_{i}^{\gamma, L O}\left(x, Q_{0}^{2}\right)+\alpha_{s}\left(Q_{0}^{2}\right) q_{i}^{(1)}\left(x, Q_{0}^{2}\right)
$$

These are substituted into the defining equation for $F_{2}^{\gamma}\left(x, Q_{0}^{2}\right)$ which is

$$
\begin{aligned}
& \frac{1}{x} F_{2}^{\gamma}\left(x, Q^{2}\right)=\left(\delta(1-x)+\frac{\alpha_{s}\left(Q^{2}\right)}{4 \pi} B_{q}(x)\right) * q_{N S}^{\gamma}\left(x, Q^{2}\right) \\
& +<e^{2}>\left(\delta(1-x)+\frac{\alpha_{s}\left(Q^{2}\right)}{4 \pi} B_{q}(x)\right) * \Sigma^{\gamma}\left(x, Q^{2}\right) \\
& +<e^{2}>\frac{\alpha_{s}\left(Q^{2}\right)}{4 \pi} B_{G}(x) * g^{\gamma}\left(x, Q^{2}\right)+\delta_{\gamma} B_{\gamma}(x),
\end{aligned}
$$

where

$$
\delta_{\gamma}=3 N_{f}<e^{4}>\frac{\alpha_{e m}}{4 \pi} .
$$

and the functions $B_{q}(x), B_{G}(x)$ and $B_{\gamma}(x)$ are the Wilson coefficient functions. $\alpha_{s}\left(Q^{2}\right)$ is now the two loop coupling constant defined by

$$
\alpha_{s}\left(Q^{2}\right) \approx \frac{4 \pi}{\beta_{0} \ln \left(Q^{2} / \Lambda^{2}\right)}\left[1-\frac{\beta_{1} \ln \left[\ln \left(Q^{2} / \Lambda^{2}\right)\right]}{\beta_{0}^{2} \ln \left(Q^{2} / \Lambda^{2}\right)}\right] .
$$


Combining these equations at $Q^{2}=Q_{0}^{2}$ with a few extra assumptions to get the singlet and non-singlet distributions enables us to extract the $q_{i}^{(1)}$ and hence NLO quark distributions [1]. Of course, to carry this out we need to specify the renormalisation scheme as the Wilson coefficients and hence the pdfs are scheme dependent. It is only in combinations such as eq.(2.14) that they have physical significance. We work in the $\overline{M S}$ scheme: expressions for the Wilson coefficients in this scheme are given in ref [30].

There is a subtlety peculiar to the photon in the $\overline{M S}$ scheme which is discussed in detail in ref. 25. 33. It arises because $B_{\gamma}$ is divergent and negative as $x \rightarrow 1\left(B_{\gamma} \propto \ln (1-x)\right)$. This means that if we put our LO input into eq.(2.14), we would get a negative $F_{2}^{\gamma}\left(x, Q_{0}^{2}\right)$ at large $x$, though the problem goes away at very large $Q^{2}$. The method outlined here eliminates this problem of negative values of $F_{2}^{\gamma}\left(x, Q_{0}^{2}\right)$ at large $x$ due to the $B_{\gamma}(x)$ term in the definition of the structure function. What we have done in imposing eq.(2.11) is essentially to add a term to the quark distributions to compensate for the $B_{\gamma}$ term. The resulting distributions are steeply rising with $x$ and are quite different from the LO ones, as we shall see.

\section{FITS TO DATA}

\section{A. Structure Function Data}

Here we fit all the data on $F_{2}^{\gamma}$ with $Q^{2} \geq 3 \mathrm{GeV}^{2}$ : we include data from PETRA 42 48, PEP 49 51], TRISTAN [52 55], and LEP1 [2,56]. The data cover a wide range of $Q^{2}$ and some have the charm subtracted out and some do not and so we must have a consistent policy over its treatment. For $Q_{0}^{2} \leq Q^{2} \leq Q_{1}^{2}=50 \mathrm{GeV}^{2}$, we use $N_{f}=3$ evolution and when we compare to data which includes charm we add to $F_{2}^{\gamma}$ the B-H estimate of charm as provided by eq.(2.6), taking $m_{c}=1.5 \mathrm{GeV}$. For $Q^{2} \geq Q_{1}^{2}$, all of the data have charm included. For these, we use a $N_{f}=4$ evolution which has been started from $Q^{2}=Q_{2}^{2}=10$ $\mathrm{GeV}^{2}$ with inputs generated by our $N_{f}=3$ evolution from $Q_{0}^{2}$ to $Q_{2}^{2}$ supplemented by the addition of the B-H term at $Q_{2}^{2}$. Throughout we keep $\Lambda_{\overline{M S}}$ fixed at $200 \mathrm{MeV}$. 
In figs.(1-4) we show the fits to the data on the $x$-distributions at various $Q^{2}$ in both $\mathrm{LO}$ and NLO. To confirm the insensitivity of structure function analyses to the gluon content of the photon we show a sample of fits corresponding to different values of $\lambda$. It can be seen that the curves are indistinguishable, except at small $x$.

In fig, 5 we show the fit to the data on

$$
\int_{0.3}^{0.8} F_{2}^{\gamma}\left(x, Q^{2}\right) d x
$$

plotted against $\ln \left(Q^{2}\right)$ for LO and NLO. The two curves agree at $Q_{0}^{2}$ but it can be argued that at higher $Q^{2}$, the NLO evolution gives a somewhat better agreement with the data. It should also be noted that our method of heavy flavour inclusion, discussed above, leads to no discontinuity in the curves when one passes the heavy flavour thresholds.

\section{B. Jets in $\gamma \gamma$ collisions}

Until relatively recently, studies of jets in $\gamma \gamma$ reactions were confined to model-dependent analyses of multiparticle distributions such as thrust. These were able to prove the existence [57.58] of the contribution from resolved photon processes [59], but were not precise enough to distinguish between different photon pdfs, though some effort was made along these lines by the later analyses [3, [4], indicating some preference for the GS [1] pdfs.

Since then data on the production of jets in appeared from the TRISTAN collaborations AMY [60] and TOPAZ [54,61]. Here we will attempt to constrain the input gluon distribution by fitting to their data on single and two-jet production. These have been shown to be somewhat sensitive to $g^{\gamma}$ in that they cannot be fitted with $g^{\gamma}=0$ and also have been used to rule out the somewhat extreme LAC3 [36] parametrisation, which, with its large gluon component at large $x$, gives much too high a cross section. Some calculations of jet production in $\gamma \gamma$ collisions in NLO have been carried out [5.66.62], which have mainly concentrated on the theoretical uncertainties, although some preliminary phenomenology has been done [6,62]. In view of the theoretical and experimental uncertainties we feel that at the moment it is only worth carrying out a LO comparison with the data. 
In this, for the photon flux we use the Equivalent Photon Approximation(EPA) 663, with parameters fixed according to the experimental conditions. We keep our quark distributions fixed at the values we obtained in subsect. IIIA, and fit the data by varying the gluon content achieved by varying the parameter $\lambda$. We find good fits to the data with $\lambda$ in the range $0.90-1.0$. We show our fits to the data in figs. 6 and 7. To illustrate our sensitivity to the gluon we also show sample fits with $g^{\gamma}=0$ and those corresponding to $\lambda=0$ and 1

\section{Jets in $\gamma p$ Collisions}

At HERA, ep interactions in the untagged case are dominated by photoproduction with the spectrum of the (mainly) real photons given by the EPA [63]. The first data on jets [64] were superseded by the first jet cross section data which enabled the direct photon reactions to be separated from the resolved photon reactions [66] and for the LAC3 [36] pdfs to ruled out as they overestimated the jet cross section 65] because of their large gluon distribution. Since then much better quality data on jet and di-jet cross sections [11, 12, 67, 68] have been published which it is hoped can be used to give information on the pdfs of the photon. These data have been confronted by NLO calculations [69,70,9] and the fits are not good [10]. What conclusions can be drawn from this is not clear at the moment as there are other

aspects of the data which are difficult to understand, for example, the energy flow out of the jet for the resolved component [71]. Multiple parton interactions have been suggested as the reason [72] but it is not clear how they can be incorporated into a NLO calculation. In a LO calculation, they give an effect in the right direction, increasing the cross section in the regions which are dominated by the resolved contribution, particularly at lower $p_{T}$, and they also help with the energy flow problem.

In view of this confusion, it seems premature to compare the results of a full NLO calculation with the data: we will simply confine ourselves to a few semi-quantitative remarks on what has been learned from the data that is relevant to pdfs. The demise of LAC3 shows that the we cannot have a large gluon distribution at large $x$. There are two other places 
where something relevant can be learnt.

The first is the fact that many pdfs overestimate the photoproduction cross section in the region of extreme negative rapidity. This is true for both single jet [69, 70, 10] and dijet [9,10] cross sections. Here the cross section is supposed to be dominated by the direct contribution which depend only on the proton pdfs which are well-known from deep inelastic scattering from protons. In direct photon processes multiple parton interactions cannot be the culprit: in fact the energy flow is well understood here. It turns out that with the NLO pdfs used initially [70, 10, 69, 70], GRV and AFG, the resolved contributions make a significant contribution here, presumably because their quark pdfs are large at large $x$, and a parton with large $x$ contributes like a direct photon, as it brings all of the photon energy into the hard subprocess. This problem goes away if the GS distributions are used both in the dijet case [9, 10] and in the single jet case [10]. This is presumably because they are relatively suppressed at large $x$, as we pointed out earlier, certainly in LO where the pdfs have physical significance, and this relative suppession is reflected in the NLO quark distributions [23]. This difference in the quark distributions does not show up in structure function analyses: there are no data at large $x$ and are unlikely to be in the future as large $x$ corresponds to $W$, the energy of the $\gamma \gamma \rightarrow$ hadrons, process being very small.

The other place is the gluon distribution of the photon which has been "extracted" by H1 [68], They used the following procedure: they constructed an $x_{\gamma}$ distribution of the dijet cross section, subtracted the contribution from direct photons to obtain the resolved cross section, and then subtracted the quark contribution from that, leaving the gluon distribution of the photon, which we plot against ours in fig. 8. There are many uncertainties associated with this procedure: it is only defined in LO, scale dependence is obviously a problem, as are multiple interactions, which are larger at small $x_{\gamma}$. Also the quark contribution subtracted was that corresponding to GRV: this is probably irrelevant in view of the other uncertainties catalogued above. However, the result is perhaps indicative that the data prefer more moderate gluon distributions such as ours and GRV. It is the extreme gluons such as LAC1 and LAC3 that have problems. 


\section{DISCUSSION}

The new GS photon distributions are many respects similar to the GS1 set of ref [1]. As we have discussed, the main improvements are; a lower starting scale for the evolution and more importantly, we have fitted to all available data on the photon structure function as well as attempted to constrain the gluon input using single and two jet data from TRISTAN. The HERA data on single and two jet production has also provided some indication that smaller quark distributions such as ours are preferred at large- $x$ and not the large distributions given by most of the other parametrisations on the market. We have attempted here to use information from all possible sources, both recent and older, experimental and theoretical, in order to constrain the parton distributions of the photon. For example the ratio $R_{2}\left(Q^{2}\right)$, discussed in the introduction, which is the ratio of the momenta carried by quarks to gluons starts at around 1 and approaches 3 as $Q^{2}$ increases. We regard this as a kind of theoretical constraint on the gluon distributions.

There are some areas where some improvements could be made in our parametrisations. Two such areas are the flavour decomposition of the structure function, and continuity in $Q^{2}$ of the quark distributions. In our treatment, the four flavour evolution is started at $Q^{2}=10 \mathrm{GeV}^{2}$ but not used except above $Q^{2}=50 \mathrm{GeV}^{2}$. At this point the charm distribution is taken as equal to that of the $u$-quark, which undoubtedly overestimates it by a considerable amount, but correspondingly, the $u$-quark distribution is reduced at this point, rendering it discontinuous there. The sum of the quark distributions, and hence $F_{2}^{\gamma}\left(x, Q^{2}\right)$ on the other hand has no discontinuity at this point. We therefore recommend that single flavour distributions, such as required for charm production in a massless framework not be calculated using our distributions.

As mentioned above, although there are no data on $F_{2}^{\gamma}\left(x, Q^{2}\right)$ at large- $x$, above $x \sim 0.8$, the recent HERA data on jet production appears to prefer smaller quark distributions in this region. At small- $x$, below $x \sim 0.05$, on the other hand we have no experimental information and hence cannot constrain the distributions here, except via the theoretically motivated 
models. It is hoped that at in the near future this situation may be remedied.

Our knowledge of the photon distributions has increased very significantly over the past few years, thanks to new experimental data from TRISTAN, LEP and HERA. The future outlook is even more optimistic, as HERA is expected to run at higher luminousity and LEP2 will soon start taking data. We anticipate that processes such as prompt photon [73], heavy flavour and lepton pair production will be measured with good statistics at these machines, allowing tighter constraints on the quark and gluon distributions of the photon.

\section{CONCLUSIONS}

We have attempted to update the GS photon distribution functions by, among other things, constraining the input gluon distributions by fitting to TRISTAN jet data. We

find that although the data is useful as an indicator of the existence of $g^{\gamma}$ and to rule out extreme distributions, it is not good enough to fix the distribution better than current VMD estimates, or other reasonable fits.

\section{ACKNOWLEDGEMENTS}

This work was supported in part by the U.S. Department of Energy, Division of High Energy Physics, Contract W-31-109-ENG-38. 


\section{REFERENCES}

[1] L.E. Gordon and J.K. Storrow, Z. Phys. C56 (1992) 307.

[2] P.Abreu et al. (DELPHI), Z. Phys. C69 (1996) 223.

[3] P.Abreu et al. (DELPHI), Z. Phys. C62 (1994) 357.

[4] P.Abreu et al. (DELPHI), Phys. Lett. B342 (1995) 402.

[5] L.E.Gordon, Nucl. Phys. B419 (1994) 25.

[6] T.Kleinwort and G.Kramer, Phys. Lett. B370 (1996)141.

[7] L.E.Gordon, Phys. Rev. D50 (1994) 6753.

[8] J.Binnewies, B.A.Kniehl and G.Kramer, DESY-96-001, MPI-PHT-96-002.

[9] M.Klasen and G.Kramer, Phys. Lett. B366 (1996) 385.

[10] G.Kramer, 'Photoproduction of Jets at HERA', J.Phys. G (to appear)

[11] M.Derrick et al. (ZEUS), Phys. Lett. B342 (1995) 417.

[12] M.Derrick et al. (ZEUS), Phys. Lett. B348 (1995) 665.

[13] J.Butterworth, 'Photon Structure as seen at HERA', Proc. 3rd Workshop on TRISTAN Physics at High Luminosity, KEK, Japan(1994).

[14] F.M.Borzumati, B.A.Kniehl and G.Kramer, Z. Phys. C59 (1993) 341.

[15] M.Greco, S.Rolli and A.Vicini, Z. Phys. C65 (1995) 277.

[16] A.C.Bawa, K.Charchula and W.J.Stirling, Phys. Lett. B313 (1993) 461.

[17] L.E.Gordon and W.Vogelsang, Phys. Rev. D52 (1995) 58.

[18] L.E.Gordon and J.K.Storrow, Z. Phys. C63 (1994) 581.

[19] E.Witten, Nucl. Phys. B120 (1977) 189. 
[20] W.A.Bardeen and A.J.Buras, Phys. Rev. D20 (1979) 166; D21 (1980) $2041 \mathrm{E}$.

[21] M.Fontannaz and E.Pilon, Phys. Rev. D45 (1992) 382; D46 (1992) 484E.

[22] R.J.De Witt et al., Phys. Rev. D19 (1979) 2046; D20 (1979) $2041 \mathrm{E}$.

[23] T.Sjöstrand, J.K.Storrow and A.Vogt LU TP 96-5 and MC-TH-96/04, to appear in'Proceedings of the Workshop on HERA Physics' Durham, England, Sept. 1995.

[24] J.K.Storrow, Proceedings of the Workshop on Two-Photon Physics at LEP and HERA, Lund, May 1994, eds. G.Jarlskog and L.Jönsson (Lund Univ., 1994) p.149.

[25] A.Vogt, Proceedings of the Workshop on Two-Photon Physics at LEP and HERA, Lund, May 1994, eds. G.Jarlskog and L.Jönsson (Lund Univ., 1994) p.141.

[26] M.Drees and R.Godbole, J. Phys. G21 (1995) 1559.

[27] J.Badier et al., Z. Phys. C18 (1983) 281; J.F.Owens and E.Reya, Phys. Rev. D17 (1978) 3003; P.Aurenche et al., Phys. Lett. B233 (1989) 517; M.Glück, E.Reya and A.Vogt, Z. Phys.C53 (1992) 651; P.J.Sutton et al, Phys. Rev. D45 (1992) 2349.

[28] M.Glück, K.Grassie and E.Reya, Phys. Rev. D30 (1984) 1447.

[29] J.H.Da Luz Vieira and J.K.Storrow, Phys. Lett. B205 (1988) 367; B219 (1989) 529E.

[30] J.H.Da Luz Vieira and J.K.Storrow, Z. Phys. C51 (1991) 241.

[31] M.Glück, E.Reya and A.Vogt, Phys. Rev. D46 (1992) 1973.

[32] P.Aurenche et al.,, Z.Phys. C56 (1992) 589.

[33] P.Aurenche, M.Fontannaz and J.P.Guillet, Z. Phys. C64 (1994) 621.

[34] G.A.Schuler and T.Sjostrand, C68 (1995) 607.

[35] M.Drees and K.Grassie, Z. Phys. C28 (1985) 451.

[36] H. Abramowicz, K. Charchula and A. Levy, Phys. Lett. B269 (1991) 458. 
[37] K.Hagiwara, M.Tanaka, I.Watanabe and T.Izubuchi, Phys. Rev. D51 (1995) 3197.

[38] W.R.Frazer and J.F.Gunion, D20 (1979) 147.

[39] M.Glück and E.Reya, Nucl. Phys. B311 (1988/9) 519.

[40] V.Budnev et al., Phys. Rep. 15C (1975) 182.

[41] E.Gotsman et al., Z. Phys. C40 (1988) 117.

[42] Ch.Berger et al. (PLUTO), Phys. Lett. B142 (1984) 111.

[43] Ch.Berger et al., Nucl. Phys. B281 (1987) 365.

[44] M.Althoff et al. (TASSO), Z. Phys. C31 (1986) 527.

[45] W.Bartel et al. (JADE), Phys. Lett. B121 (1983) 203.

[46] W.Bartel et al. (JADE),Z. Phys. C24 (1984) 231.

[47] H.J.Behrend et al. (CELLO), Phys. Lett. B126 (1983) 391.

[48] H.J.Behrend et al. (CELLO), contributed to the 25th Int. Conf. on HEP, Singapore, 1990.

[49] H.Aihara et al. (TPC), Phys. Rev. Lett. 58 (1987) 97.

[50] H.Aihara et al. (TPC), Z. Phys. C34 (1987) 1.

[51] J.Steinman (TPC), UCLA report no. UCLA-HEP-004(1988)

[52] T.Sasaki et al., Phys. Lett. B252 (1990) 491.

[53] S.K.Sahu et al., Phys. Lett. B346 (1995) 208.

[54] K.Muramatsu et al. (TOPAZ), Phys. Lett. B332 (1994) 477.

[55] S.Uehara et al. (VENUS) Private communication.

[56] R.Akers et al. (OPAL), Z. Phys. C61 (1994) 199. 
[57] A.Bäcker, Proc. 7th Intern. Workshop on Two-Photon Collisions (Paris, 1986), A.Courau, P.Kessler (eds.), p.101, Singapore: World Scientific 1986.

[58] Ch.Berger et al. (PLUTO), Z. Phys. C33 (1987) 351; H.J.Behrend et al. (CELLO), Z. Phys. C51 (1991) 365; R.Tanaka et al. (AMY), Phys. Lett. B277 (1992) 215; D.Buskulic et al (ALEPH), Phys. Lett. B313 (1993) 509.

[59] M.Drees and R.Godbole, Nucl. Phys. B339 (1990) 355; S.J.Brodsky et al, Phys. Rev. Lett. 41 (1978) 672 and Phys. Rev. D19 (1979) 1418; K.Kajantie and R.Raito, Nucl. Phys. B159 (1979) 528.

[60] B.J.Kim et al. (AMY), Phys. Lett. B325 (1994) 248.

[61] H.Hayahashi et al. (TOPAZ), Phys. Lett. B314 (1993) 149.

[62] P.Aurenche et al., Prog. Theor. Phys. 92 (1994) 175.

[63] F. von Weizsäcker, Z. Phys. 88 (1934) 612. ;E.J. Williams,Phys. Rev. 45 (1934) 729.

[64] T.Ahmed et al. (H1), Phys. Lett. B297 (1992) 205; M.Derrick et al. (ZEUS), Phys. Lett. B297 (1992) 404.

[65] I.Abt et al. (H1), Phys. Lett. B314 (1993) 436.

[66] M.Derrick et al. (ZEUS), Phys. Lett. B322 (1994) 287.

[67] S.Aid et al. (H1), Z. Phys. C70 (1996) 17.

[68] T.Ahmed et al. (H1), Nucl. Phys. B445 (1995) 195.

[69] P.Aurenche, J.-Ph.Guillet, and M.Fontannaz, Phys. Lett. B338 (1994) 98.

[70] M.Klasen, G.Kramer, and S.G.Salesch, Z. Phys. C68 (1995) 113.

[71] S.J.Maxfield, To appear in 'Proc. of the Workshop on HERA Physics' Durham, England, Sept. 1995. 
[72] J.Butterworth, J.R.Forshaw, and M.Seymour, CERN-TH/95-82.

[73] L.E.Gordon and J.K.Storrow, ANL-HEP-PR-96-32 and MC-TH-96/15. 


\section{Figure Captions}

Fig. 1: Fits to PETRA data on the photon stucture function.

Fig. 2: Fits to PEP data on the photon structure function.

Fig. 3: Fits to TRISTAN data on the photon structure function. At $Q^{2}=390 \mathrm{GeV}^{2}$, in the second graph the solid curve is for the choice $\lambda=2.0$ (see text).

Fig. 4: Fits to LEP1 data on the photon structure function. At $Q^{2}=12 \mathrm{GeV}^{2}$, in the second graph, the dot-dashed curve is for the choice $\lambda=0$.

Fig. 5: $Q^{2}$ dependence of the structure function integrated over the region $0.3 \leq x \leq 0.8$.

Fig. 6: Fits to AMY data on $\gamma \gamma \rightarrow$ jets for two choices of $\lambda$ and for $g^{\gamma}=0$; (a) fits to the $p_{T}$ distribution of the single-jet data (b) fits to the rapidity distribution of the single-jet data and (c) fits to the $p_{T}$ distribution of the two-jet data.

Fig. 7; Fits to TOPAZ (a) single-jet and (b) two-jet data.

Fig. 8; Comparison of the GS(HO), GS(LO) and GS(I) and GS(II) LO [1] gluon distributions with the gluon distribution extracted by the H1 Collaboration 68. 


\section{FIGURES}
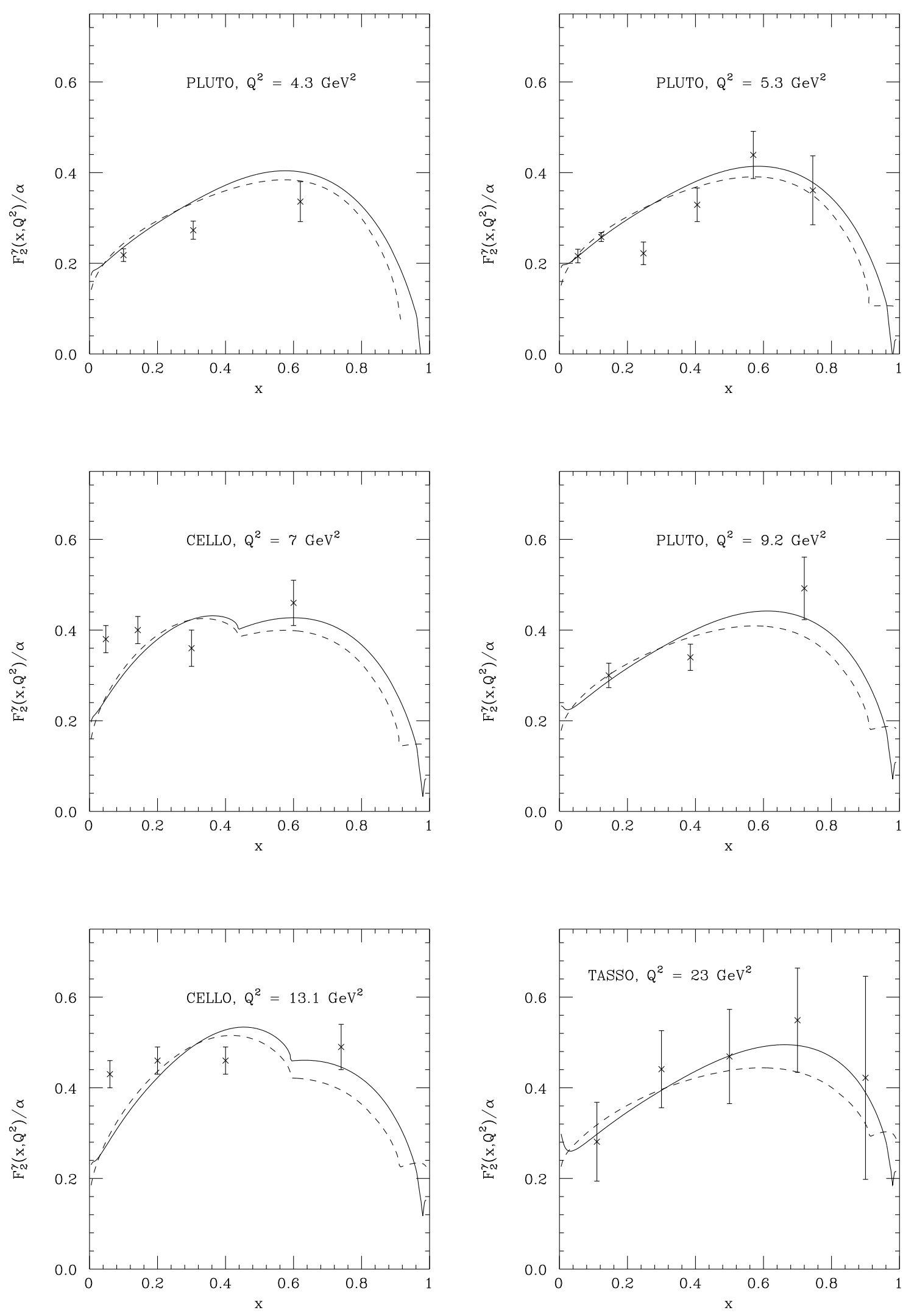

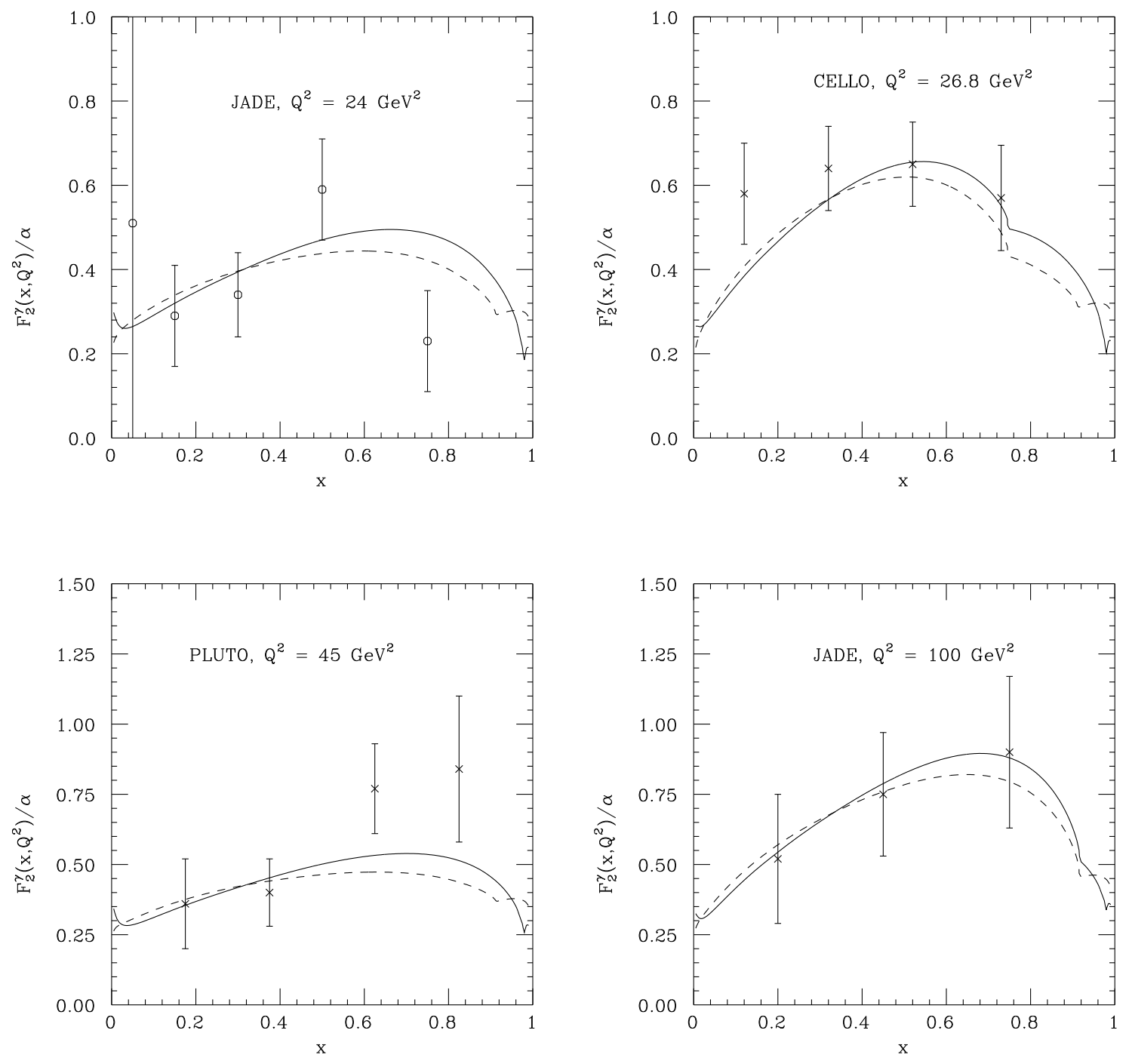

Fig. 1. 

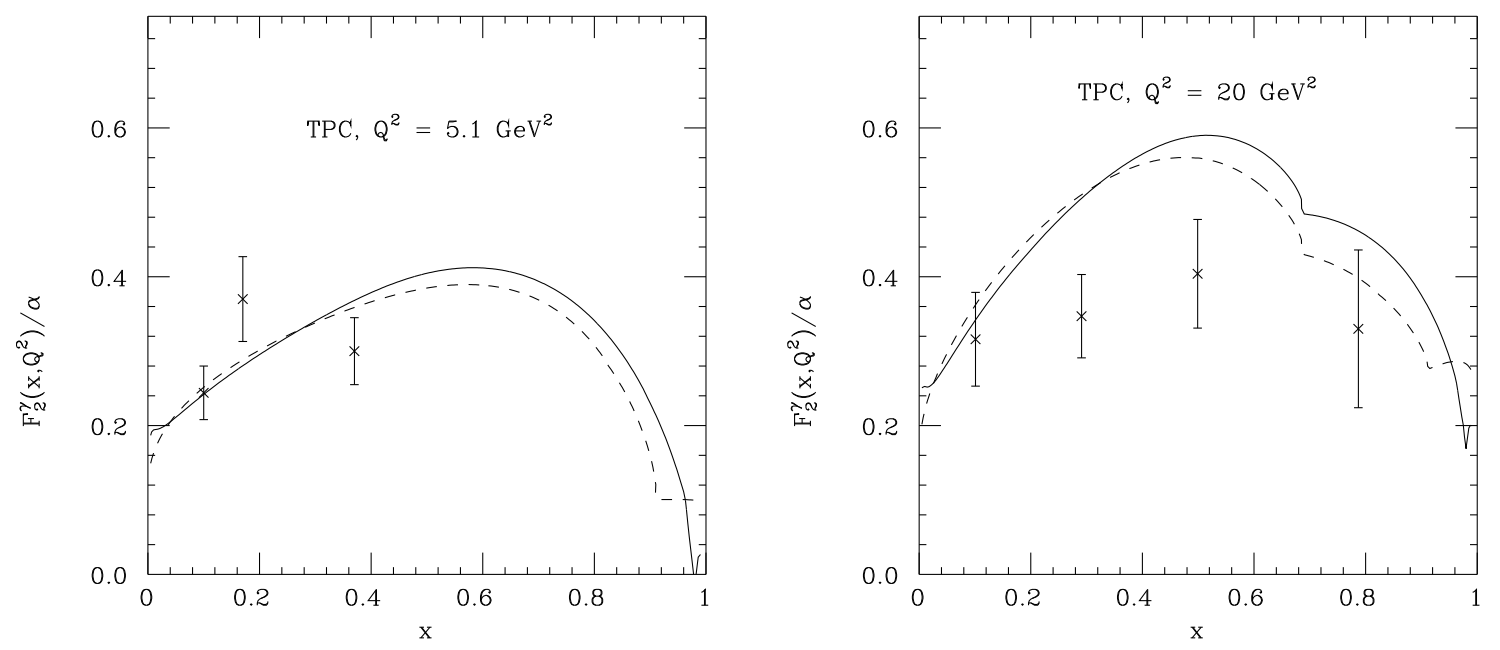

Fig. 2. 

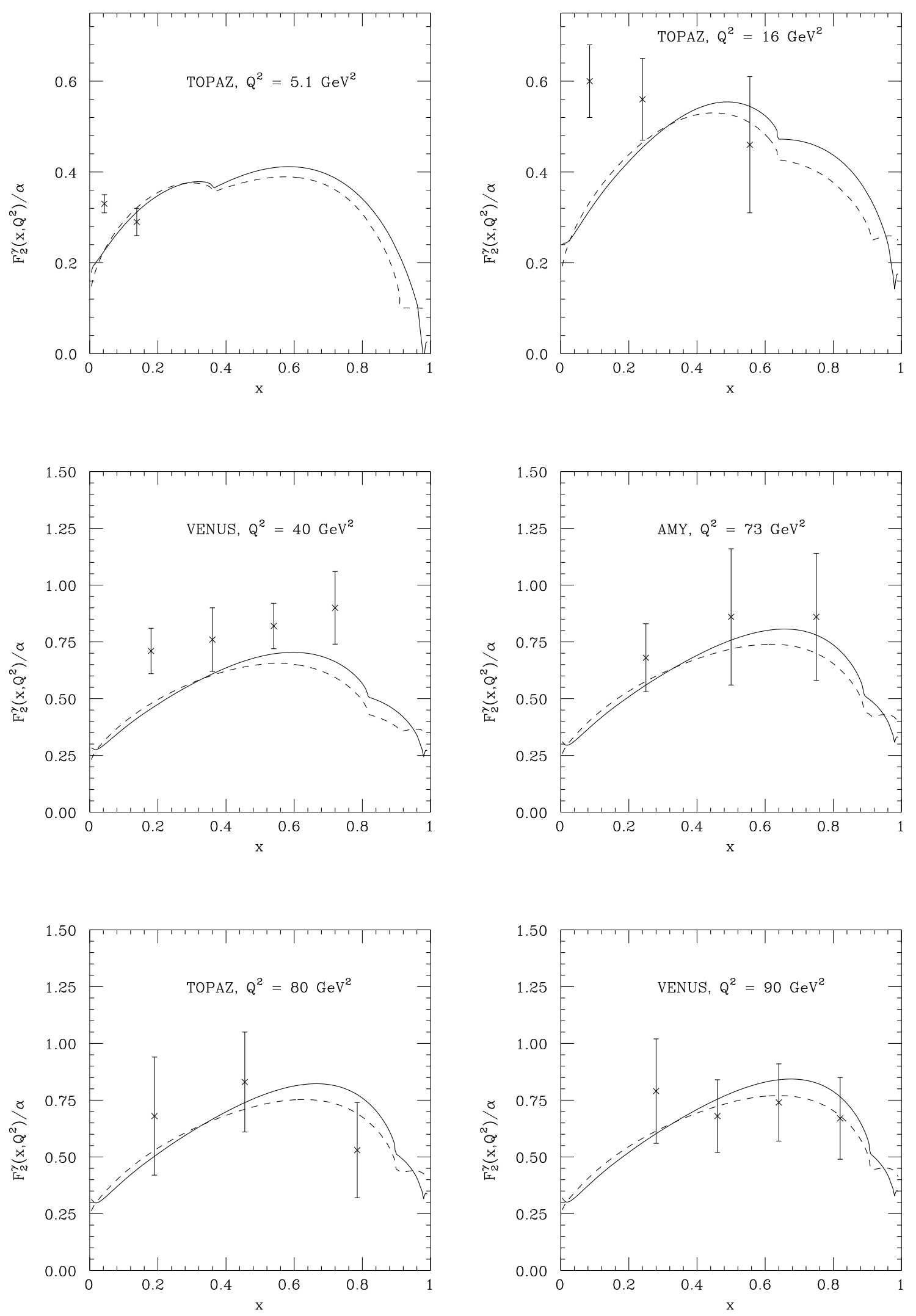

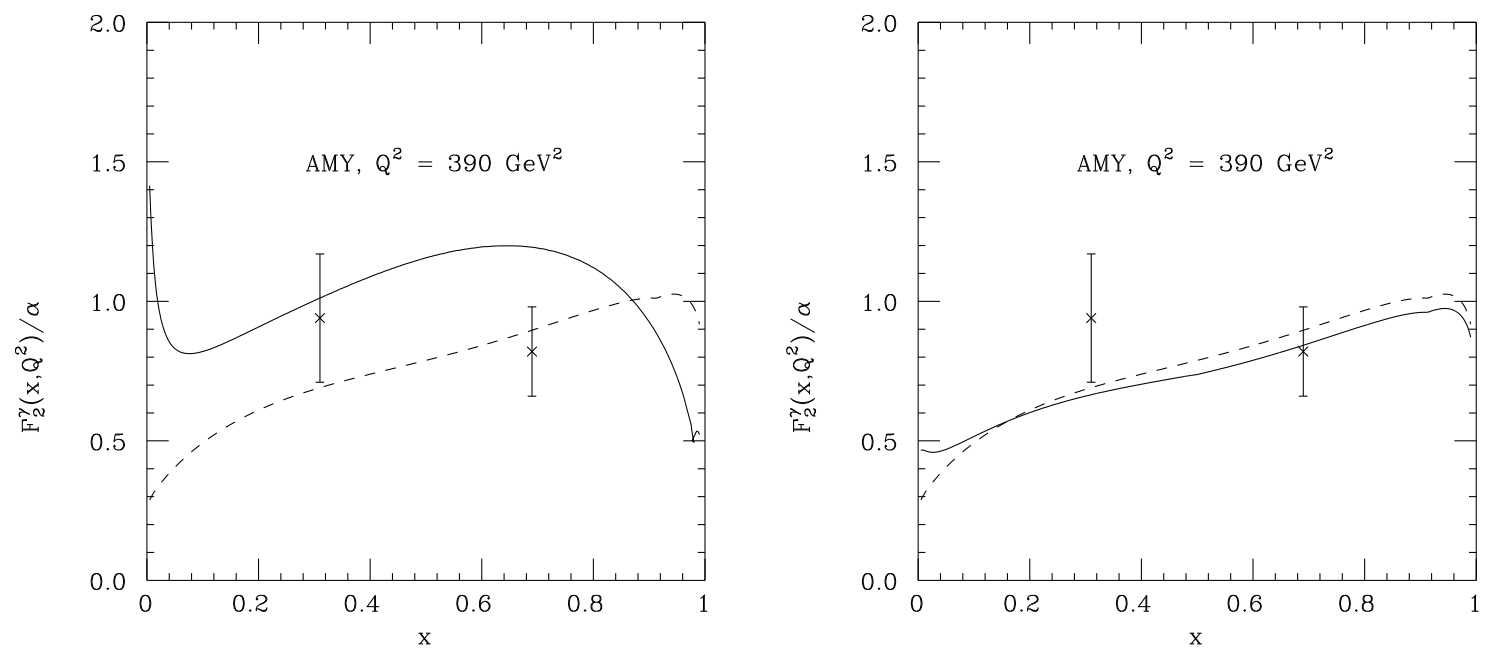

Fig. 3. 

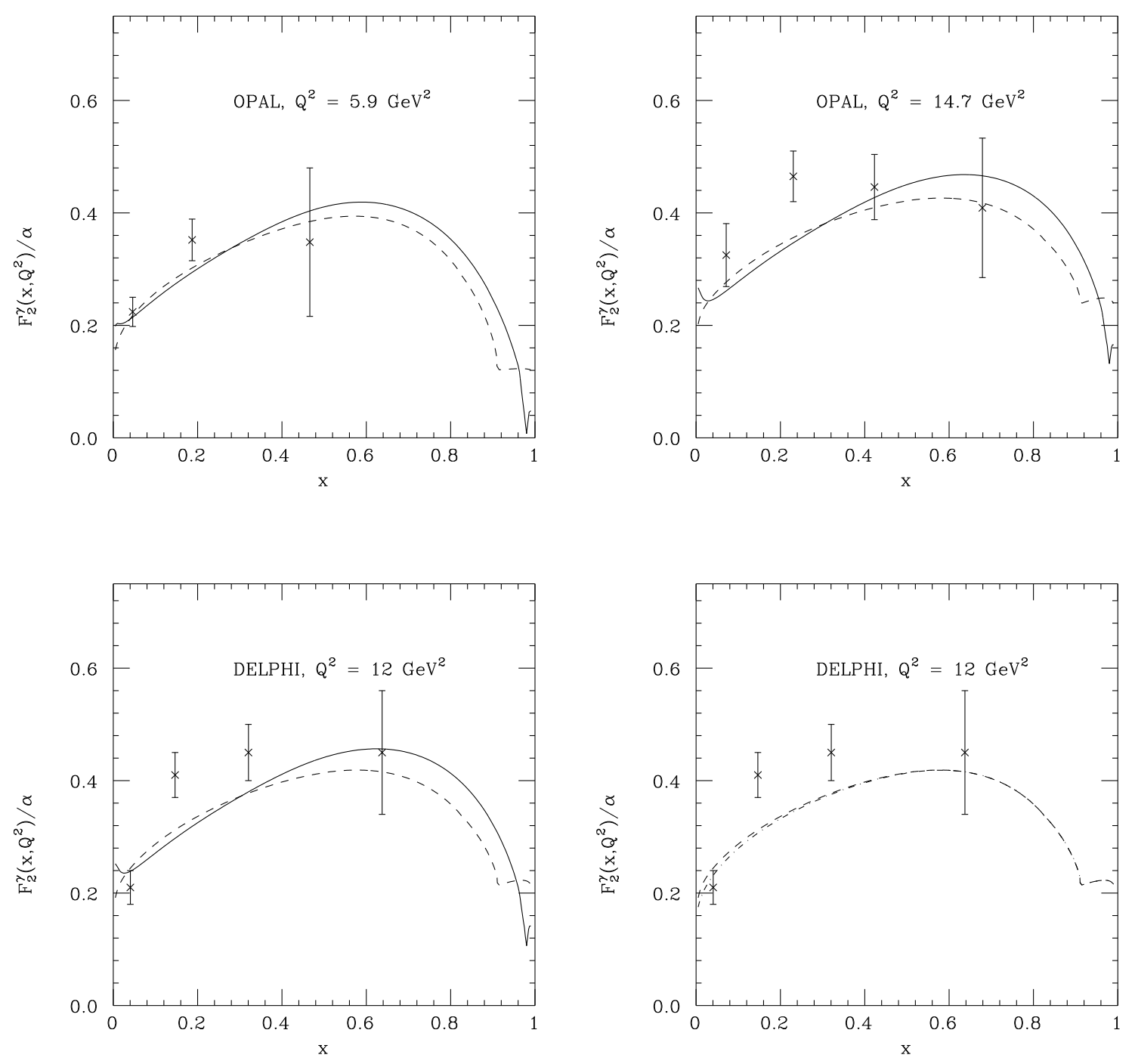

Fig. 4. 


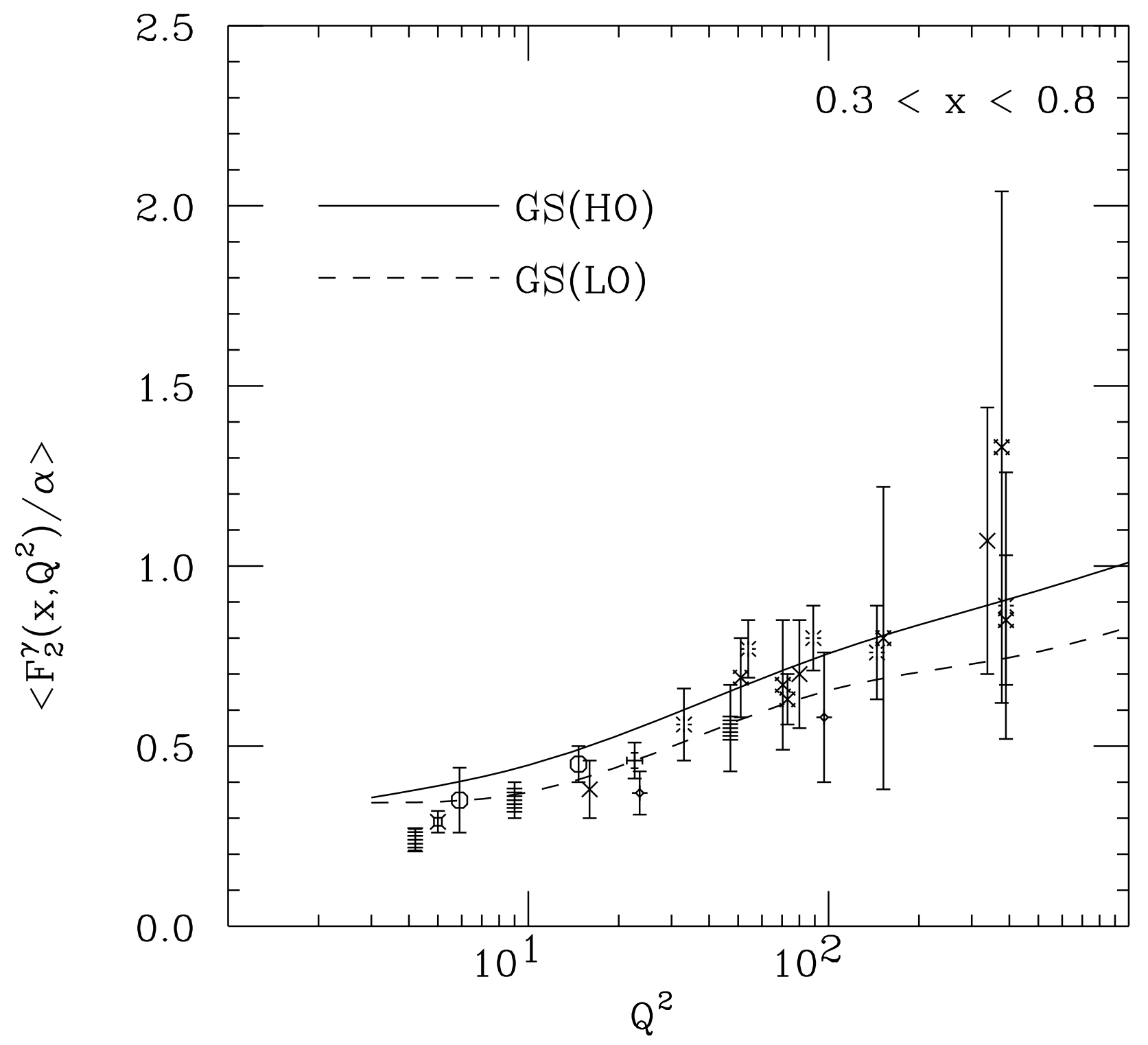

Fig. 5 


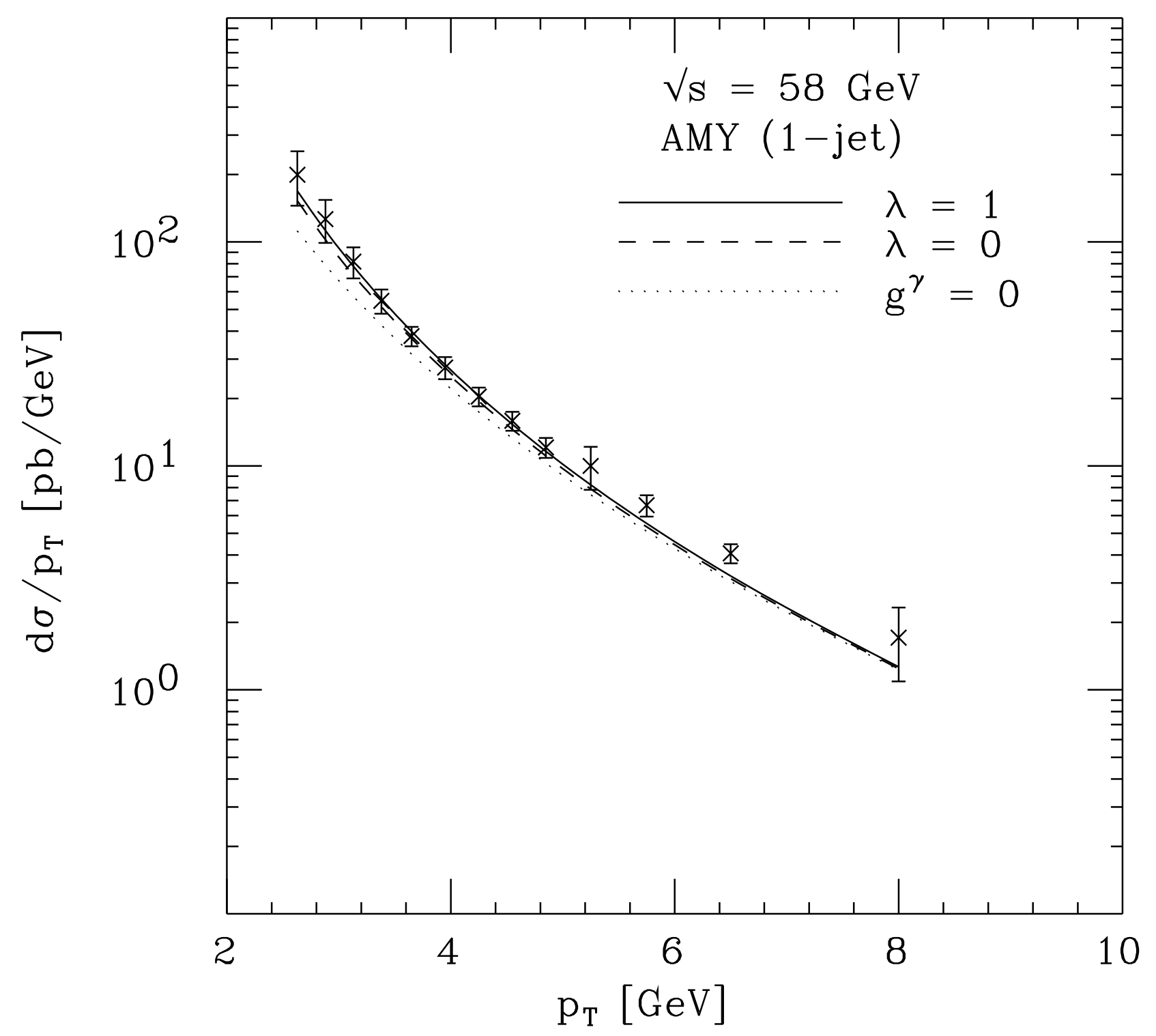

Fig. 6a 


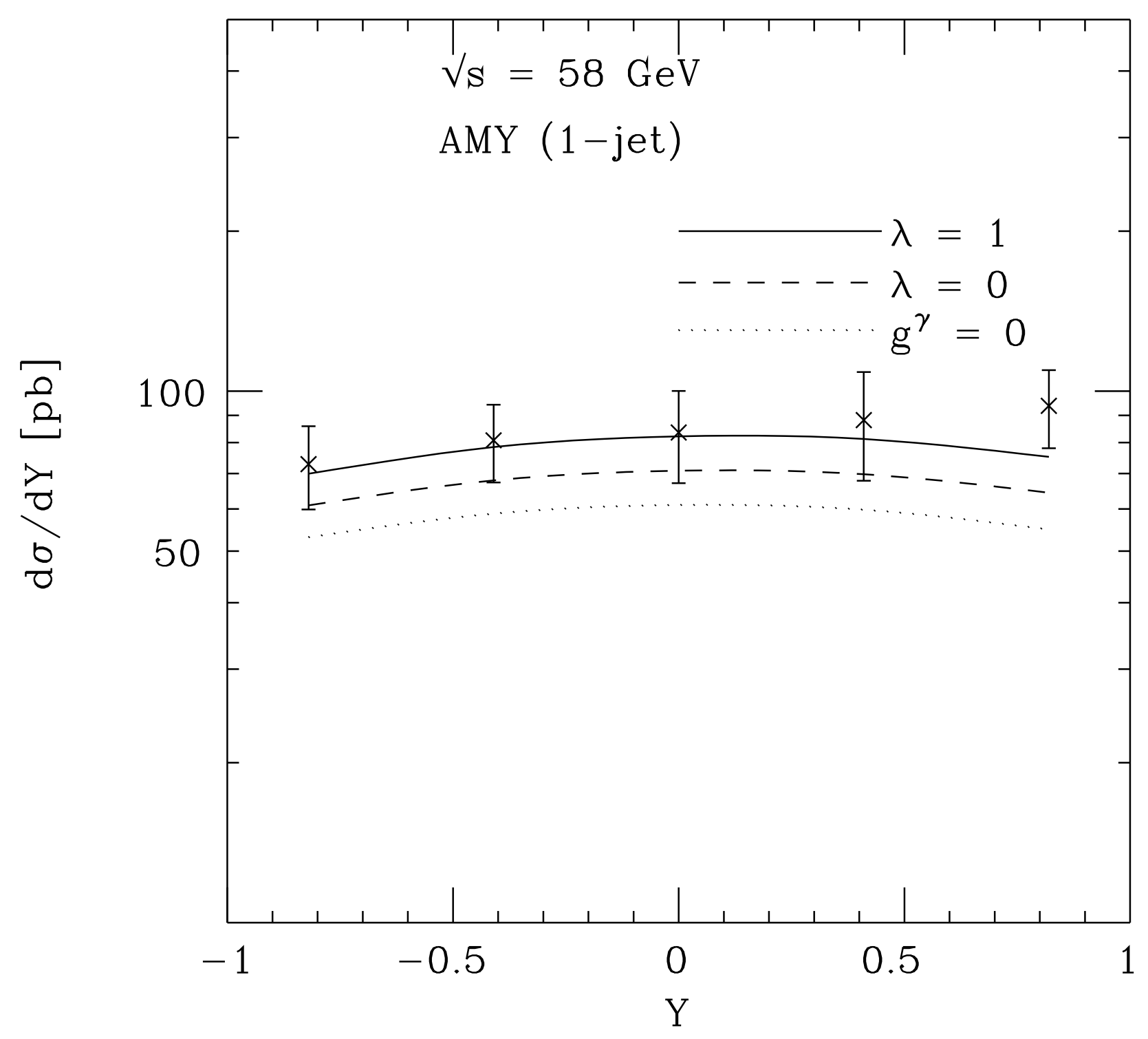

Fig. 6b 


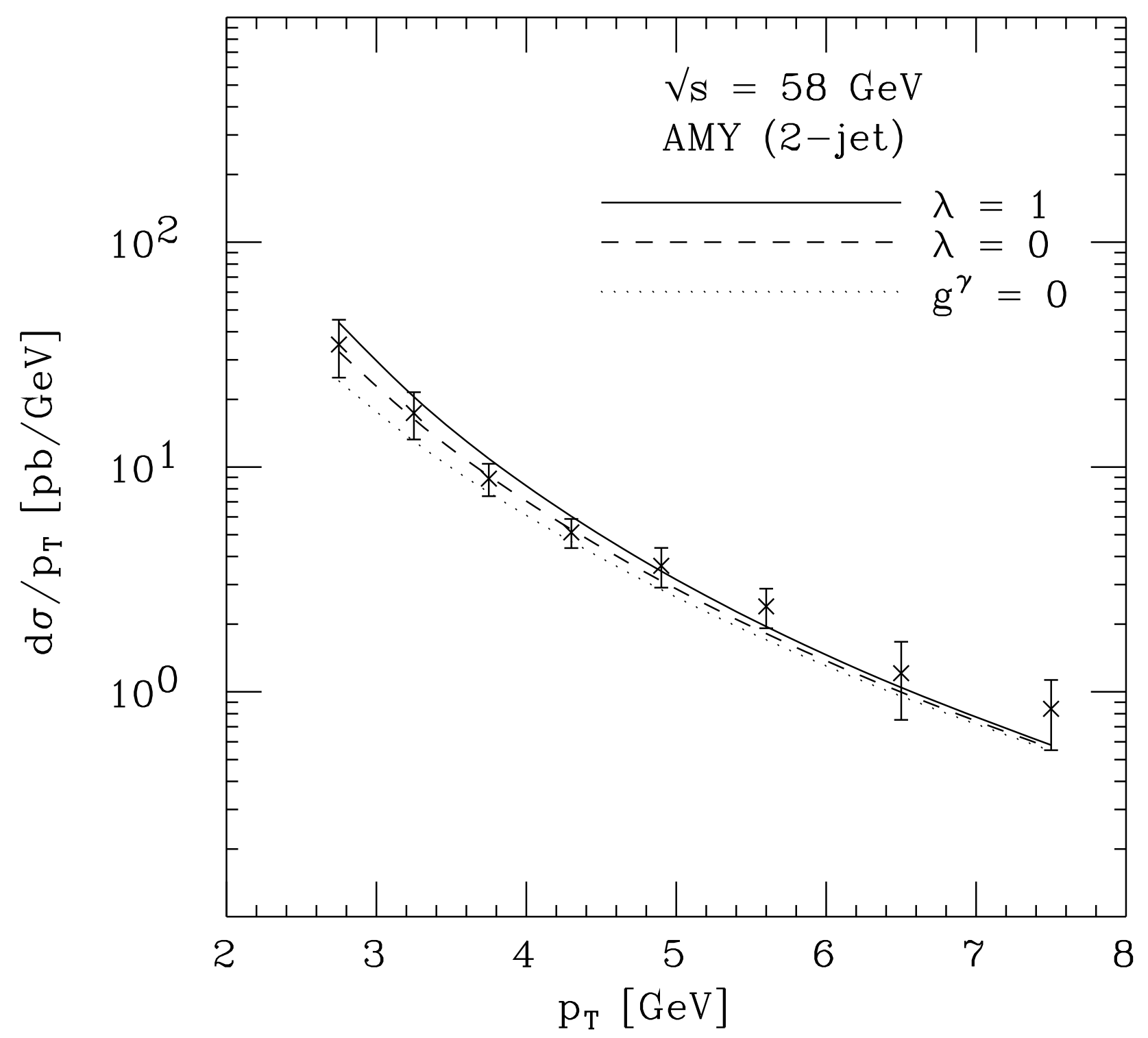

Fig. 6c 


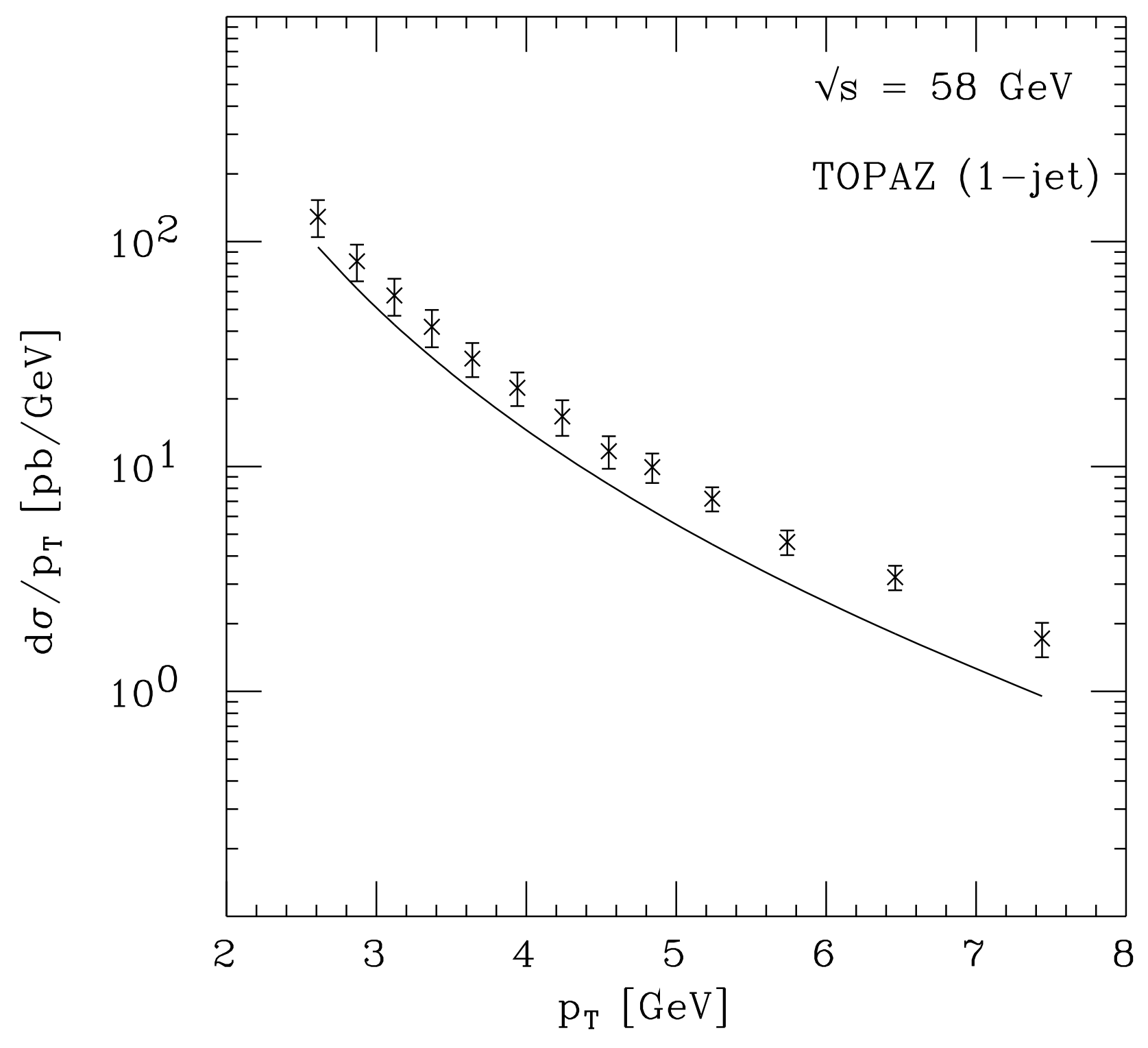

Fig. $7 a$ 


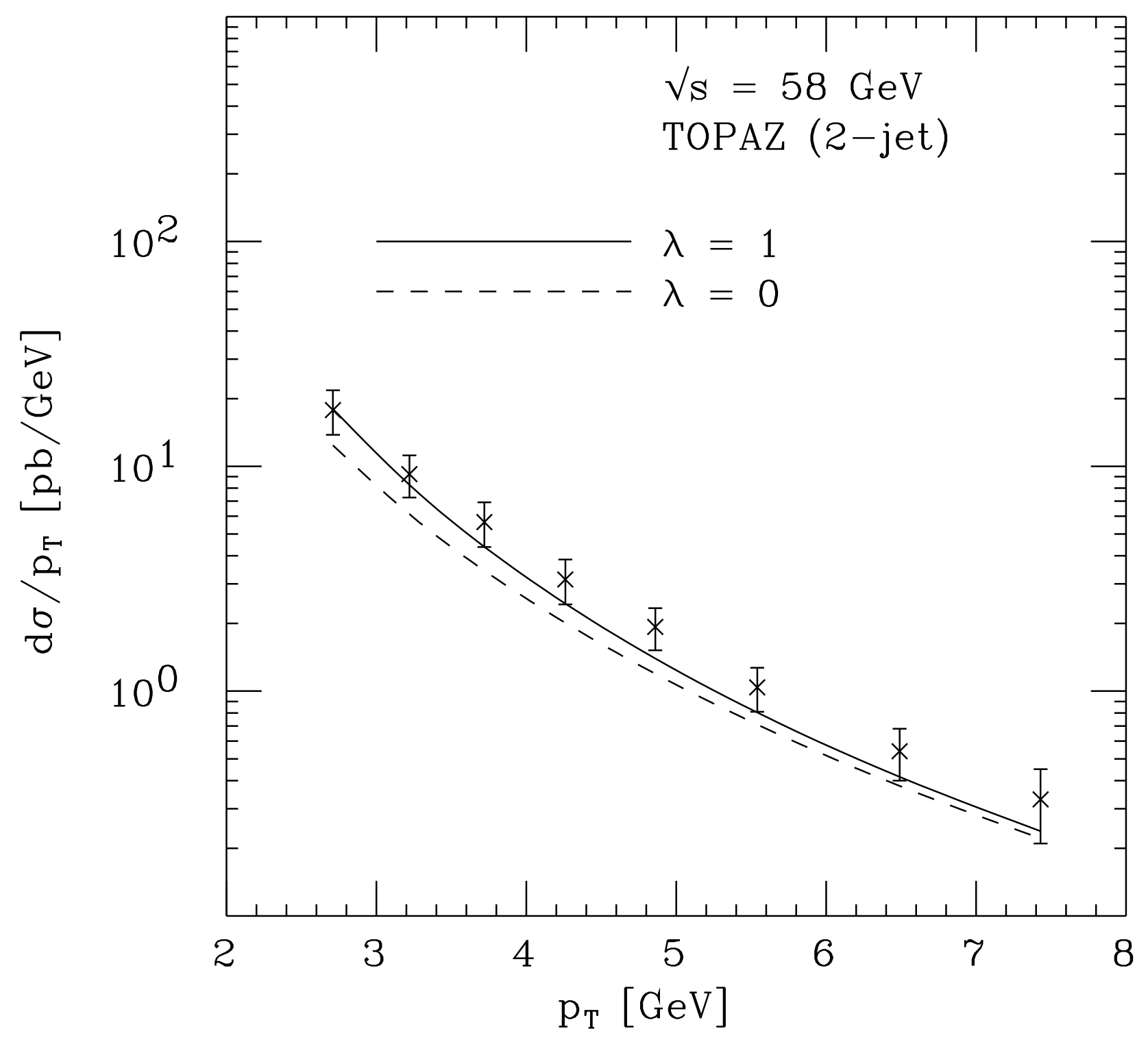

Fig. 7b 


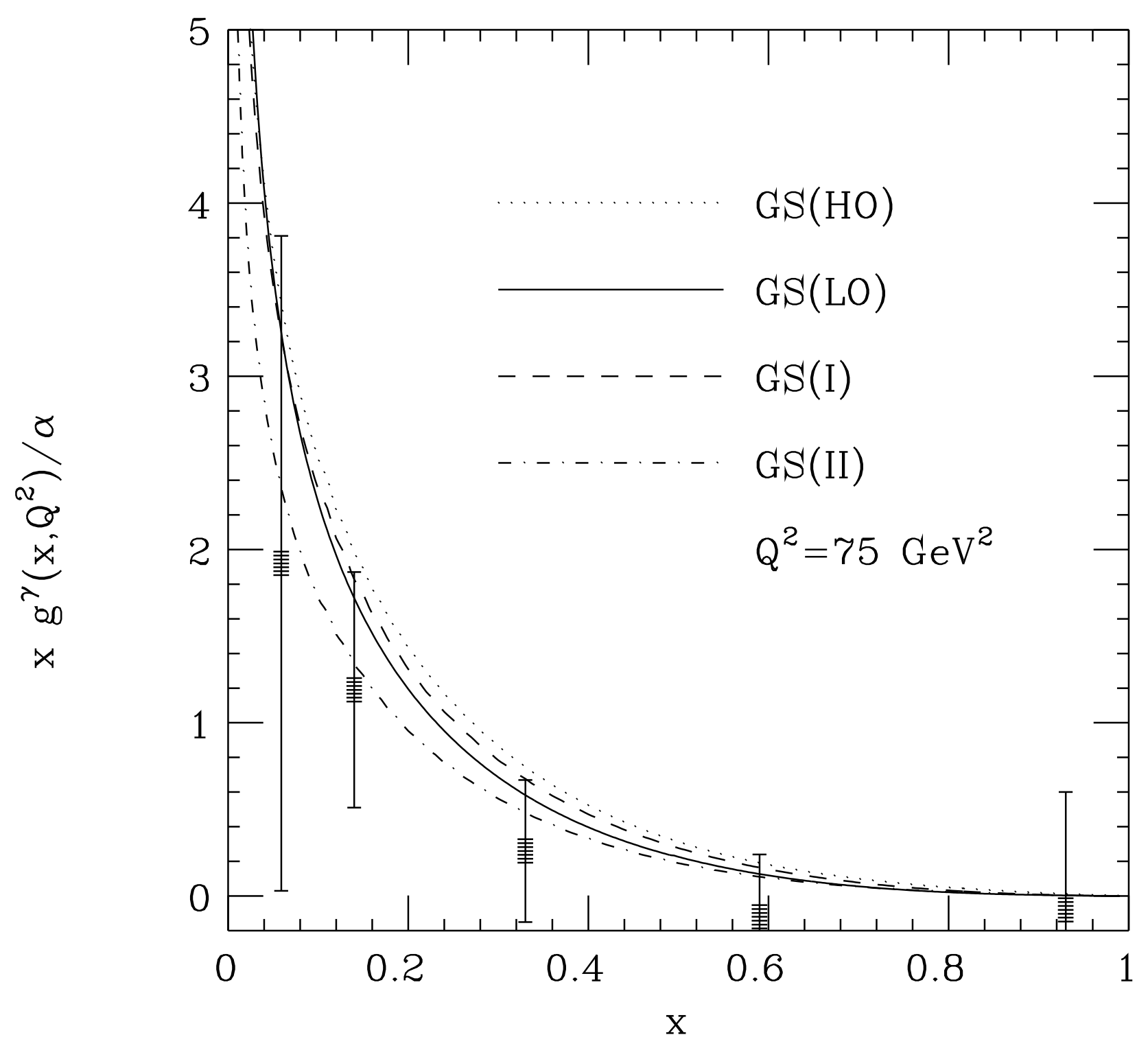

Fig.8 